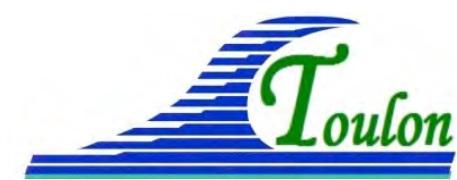

XIV $V^{e ̀ m e s}$ Journées Nationales Génie Côtier - Génie Civil

Toulon, 29 juin au $1^{\text {er }}$ juillet 2016

DOI:10.5150/jngcgc.2016.055 (C) Editions Paralia CFL

disponible en ligne - http://www.paralia.fr - available online

\title{
Simulation bidimensionnelle d'une hydrolienne à axe vertical dans un courant marin
}

\section{Zaïd HAMMOUDI ${ }^{1}$, Rafik ABSI ${ }^{2}$, Ikram EL ABBASSI ${ }^{{ }^{*}}$, Abdel Moumen DARCHERIF ${ }^{1^{* *}}$}

1. ECAM-EPMI, 13 Boulevard de l'Hautil, 95092 Cergy Pontoise, France.

* LR2E-lab, ** Quartz-lab (EA 7393)

zaid.hammoudi@hotmail.fr ; i.elabbassi@ecam-epmi.fr ; m.darcherif@ecam-epmi.fr

2. EBI, EBInnov-lab, 13 Boulevard de l’Hautil, 95092 Cergy Pontoise, France.

r.absi@hubebi.com

\section{Résumé :}

Cette étude porte sur les hydroliennes à axe vertical. Nous avons introduit leur mode de fonctionnement ainsi que les paramètres les caractérisant. La vitesse spécifique ou Tip Speed Ratio (TSR), est le rapport entre la vitesse périphérique des pales et la vitesse de l'écoulement. Afin de mieux comprendre son importance, nous avons effectué des simulations bidimensionnelles avec ANSYS Fluent. Nous avons étudié le comportement d'une hydrolienne à axe vertical de type Darrieus avec deux types de profil de pales : le NACA0018 puis le NACA 4415. Nous avons aussi abordé l'interaction entre deux hydroliennes alignées axialement dans le sens de l'écoulement. Nous avons indiqué les étapes suivies pour la création d'une géométrie et un maillage en vue d'appliquer la technique du maillage glissant avec ANSYS Design Modeler, Meshing et Fluent.

Mots-clés : Hydroliennes, Vitesse spécifique, Puissance, Profil de pales, Ansys Fluent.

\section{Introduction}

L’énergie des combustibles fossiles et du nucléaire représente plus de $80 \%$ de l'énergie utilisée dans le monde. Ces sources sont faciles à exploiter mais le problème majeur est qu'elles ne sont pas inépuisables, et aussi qu'elles provoquent des problèmes environnementaux, comme l'effet de serre et les déchets nucléaires. La plupart des pays ont déjà commencé une transition vers des énergies propres et renouvelables pour assurer leur avenir.

Les énergies renouvelables peuvent être classées en cinq familles : le solaire, l'éolien, la biomasse, la géothermie et l'hydraulique ou l'hydroélectrique. Dans ce travail, c'est cette dernière qui nous intéresse et plus précisément l'énergie des courants marins ou fluviaux. Celle-ci représente un énorme potentiel d'énergie durable et prédictible, qui est pour le moment peu exploitée à travers le monde. On estime le potentiel de l'énergie hydrolienne totale dans le monde à environ 3 TW dont 1 TW dans des zones accessibles pour l'installation de dispositifs de captage de cette énergie (TWIDEL \& WEIR, 2006). 


\section{Thème 5 - Énergies et ressources marines}

Pour capter cette énergie cinétique, on peut utiliser plus de 50 dispositifs (EDENHOFER et al., 2011 ; BOSSARD, 2012), plus particulièrement des machines dérivées des technologies éoliennes, appelés hydroliennes (BEHRENS et al., 2012), immergées le long des côtes ou dans les cours d'eau, ou suspendues à la surface de l'eau (KIRKE, 2011). Il existe principalement deux types d'hydroliennes selon le positionnement de leur axe de rotation par rapport au courant : celles avec un axe horizontal et celles à axe vertical. Compte tenu de l'intérêt que présente cette dernière, des études ont été réalisées pour mieux comprendre leur fonctionnement (MARSH et al., 2013 ; MARSH et al., 2015 ; MENCHACA ROA, 2011 ; HALL, 2012).

Le fonctionnement des hydroliennes est complexe. Une étude numérique globale nécessite de prendre en compte l'interaction fluide-structure. Dans la présente étude cet aspect n'est pas considéré, nous n’abordons que la partie écoulement sans toucher à l'étude mécanique de la structure des pales. Plusieurs logiciels de simulation CFD (Computational Fluid Dynamics) peuvent être utilisés, comme ANSYS CFX et Fluent (MENCHACA ROA, 2011 ; MARSH et al., 2013). Ce dernier a été utilisé pour les simulations effectuées. Ce travail porte uniquement sur les hydroliennes verticales. Nous avons introduit leur mode de fonctionnement en général, défini les différents paramètres les caractérisant et les modèles de turbulence qu'on peut utiliser pour les simulations. Enfin, des simulations avec différents paramètres et géométries ont été menées.

\section{Généralités sur les hydroliennes}

\subsection{Principe de fonctionnement}

Le principe consiste à capter l'énergie cinétique du courant et la transformer en énergie électrique en faisant tourner une turbine. Chaque hydrolienne est composée d'une base qui permet de la fixer au fond marin ou la suspendre sous la surface de l'eau et d'un rotor qui tourne sous l'effet du courant le traversant. Ce rotor entraîne alors un alternateur qui transforme l'énergie mécanique en énergie électrique.

\subsection{Puissance maximale extraite : Loi de Betz}

Toutes les machines tournantes sous l'effet d'un fluide sont soumises à la loi de Betz, une loi qui définit la puissance maximale qu'on peut extraire de ce fluide traversant une turbine d'une section $S$. La puissance extraite de l'eau par le rotor est donnée par (GROUPE 12A ECP, 2005) :

$P=\frac{1}{2}(\rho S v)\left(v_{1}^{2}-v_{2}^{2}\right)$

avec : $v_{1}$ et $v_{2}$ sont respectivement les vitesses en amont et en aval de l'hydrolienne, $\rho$ la masse volumique de l'eau et $v=\frac{1}{2}\left(v_{1}+v_{2}\right)$. 


\section{XIV èmes Journées Nationales Génie Côtier - Génie Civil \\ Toulon, 29 juin au $1^{\text {er }}$ juillet 2016}

Par ailleurs, la puissance totale $P_{0}$ d'un flux d'eau non perturbé traversant une surface similaire $S$ sans la présence d'un rotor qui le ralentit, est :

$P_{0}=\frac{1}{2} \rho S v_{1}^{3}$

Le rapport entre ces deux puissances est alors de :

$\frac{P}{P_{0}}=\frac{1}{2}\left(1-\left(\frac{v_{2}}{v_{1}}\right)^{2}\right)\left(1+\left(\frac{v_{2}}{v_{1}}\right)\right)$

La courbe de l'équation (3) atteint son maximum pour $v_{2} / v_{1}=1 / 3$, la valeur maximale est $P / P_{0}=16 / 27$.

\subsection{Vitesse spécifique ou Tip Speed Ratio}

La vitesse spécifique, appelée aussi paramètre d'avance, notée TSR pour Tip Speed Ratio, est le rapport entre la vitesse périphérique des pales et la vitesse de l'écoulement : $T S R=\lambda=\frac{\omega R}{v_{\infty}}$

avec $\omega$ la vitesse de rotation, $R$ le rayon de l'hydrolienne et $v_{\infty}$ la vitesse de l'écoulement loin de l’hydrolienne.

\subsection{Coefficients de puissance et de moment}

On introduit le coefficient de moment comme suit :

$C_{M}=\frac{M_{z}}{\frac{1}{2} \rho(H \phi) R v_{\infty}^{2}}$

avec $M_{z}$ le couple suivant l'axe de rotation $\overrightarrow{o z}, S=H \phi$, $H$ la hauteur de l'hydrolienne, $\phi$ le diamètre de l'hydrolienne.

Le coefficient de puissance $C_{P}$ est le rapport entre la puissance tirée de l'eau par l’hydrolienne et la puissance apportée par l'écoulement. IL s'écrit :

$C_{P}=\frac{P}{\frac{1}{2} \rho(H \phi) v_{\infty}^{3}}$

Nous avons aussi la relation entre la puissance et le moment :

$P=M_{z} \omega$

À partir des relations précédentes, on peut déduire que :

$C_{P}=\lambda C_{M}$

\section{Simulations}

Nous commençons par valider nos simulations par comparaison avec celles de la MENCHACA ROA, (2011) qui concernent une hydrolienne libre à axe vertical de type Darrieus (figure 1, en haut à gauche) avec des pales basées sur le profil NACA0018. Ceci permet de sélectionner le maillage le plus adéquat. Nous allons donc effectuer des simulations bidimensionnelles d'une hydrolienne verticale dans un milieu infini (figure 1 , en haut à droite). Nous testons par la suite un autre profil de pale à savoir le profil NACA 4415, pour pouvoir comparer leurs performances. Enfin, nous allons aborder l'interaction entre hydroliennes. Les simulations sous ANSYS Fluent (ANSYS INC, 


\section{Thème 5 - Énergies et ressources marines}

2013), nécessitent d'abord de créer la géométrie et le maillage. Pour cela nous avons utilisé Design Modeler pour la géométrie et Meshing pour le maillage. La méthodologie suivie est expliquée ci-après (HAMMOUDI, 2015).
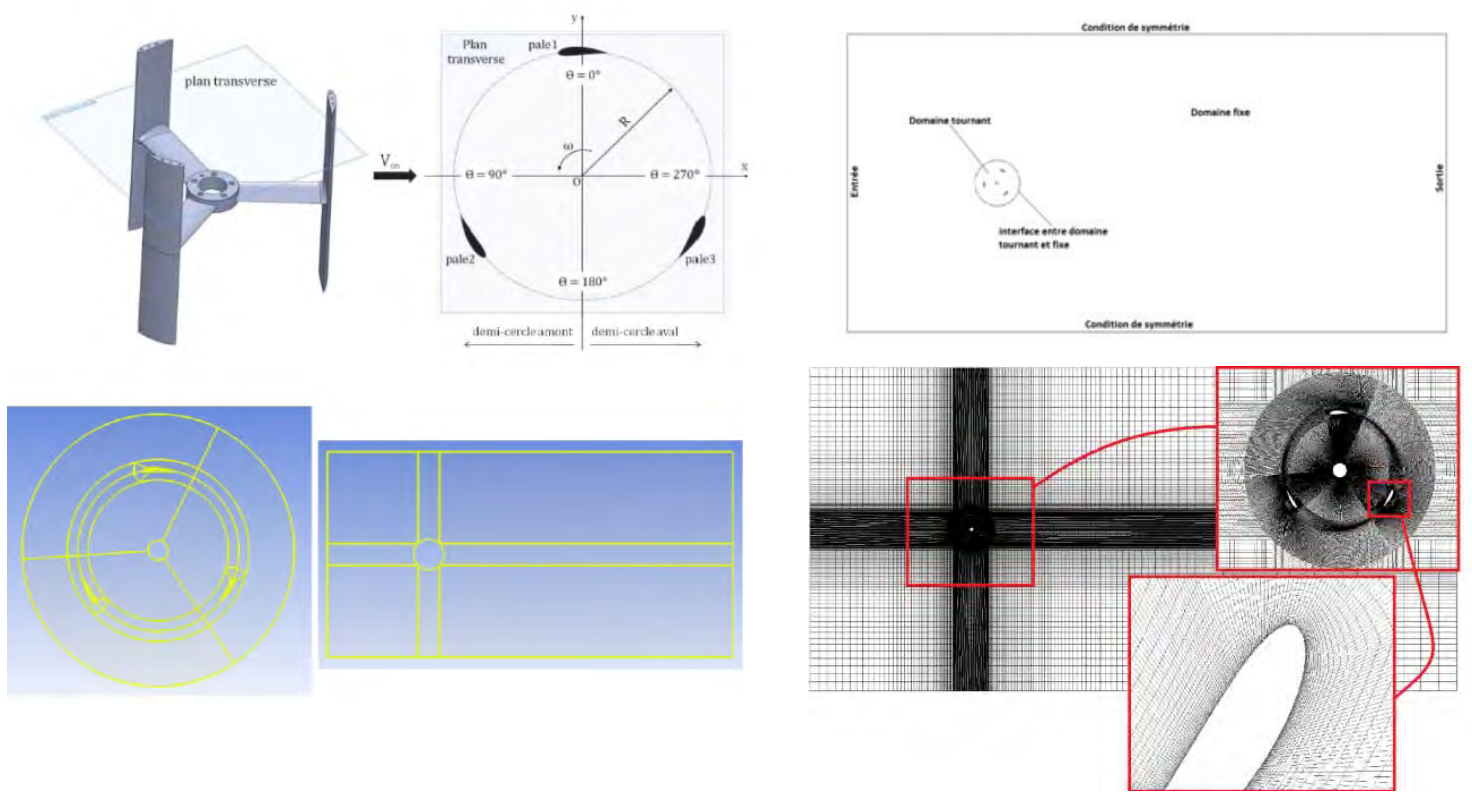

Figure 1. Géométrie et maillage.

En haut à gauche, vue 3D de l'hydrolienne et sa section $2 D$ utilisée pour les calculs. En haut à droite, géométrie d'une hydrolienne verticale dans un milieu infini. En bas à gauche, corps filaires. En bas à droite, maillage structuré.

\subsection{Géométrie}

La géométrie est constituée de deux domaines, l'un tournant, de section circulaire comportant les trois sections horizontales des pales de l'hydrolienne ainsi que son axe et, l'autre fixe représentant le milieu qui entoure l'hydrolienne. Entre les deux, nous avons une interface appelée "sliding mesh".

L'hydrolienne utilisée a un diamètre $\emptyset=(2 \mathrm{R})=0.175 \mathrm{~m}$, un axe de diamètre $d=0.022$ $\mathrm{m}$, une hauteur $h=0.175 \mathrm{~m}$, une corde de pales $C=0.032 \mathrm{~m}$ et une forme de pale qui change en fonction du profil choisi. La géométrie est réalisée avec Design Modeler.

\subsection{Maillage}

Le maillage est réalisé à l'aide du logiciel Meshing intégré dans ANSYS Workbench. Géométrie déjà partagé en corps filaire, il ne reste qu'à créer des dimensionnements sur chaque arête, des inflations autour des parois des pales et de l'axe de rotation et enfin appliquer la méthode de maillage de faces pour créer un maillage en quadrilatères (figure 1, en bas à droite). Au final il reste à ajouter les sélections nommées pour les limites et aussi l'interface entre partie rotative et fixe (ANSYS INC, 2013). 


\section{XIV ${ }^{\text {èmes }}$ Journées Nationales Génie Côtier - Génie Civil \\ Toulon, 29 juin au $1^{\text {er }}$ juillet 2016}

\subsection{Paramètres utilisés sous Fluent}

Une fois le maillage créé, il ne reste qu’à l’importer sous Fluent et régler les différents paramètres avant de lancer le calcul. Tout d'abord il faut créer une interface entre la partie rotative et la partie fixe : "Mesh Interface". Ensuite on pourra procéder aux autres réglages comme suit: (i) sélectionner "transient" dans "general" pour passer en instationnaire, (ii) choisir le modèle de turbulence, k-omega (2 équations) dans "Model" et SST dans "k-omega model", (iii), dans "Cell Zone Conditions", donner la rotation voulue pour la zone rotative en cochant la case "Mesh Motion" et (iv) choisir l'axe de rotation et la vitesse angulaire. Pour la définition des conditions aux limites dans "Boundary Conditions", on choisit "symmetry" pour les deux frontières horizontales du domaine, et "wall" pour les trois pales. Pour les paramètres d'entrée, on retient: "velocity inlet", vitesse égale à $2.3 \mathrm{~m} / \mathrm{s}$, intensité de turbulence $I=3 \%$, longueur caractéristique $l=0.002$ comme grandeurs de turbulence. Enfin en paramètre de sortie comme "pressure outlet", on a les mêmes paramètres de turbulence qu'à l'entrée. Pour le calcul du coefficient de moment $C_{M}$ donné par l'équation (5), Fluent utilise des valeurs de références, qu'on doit insérer dans "reference values"; ces valeurs sont la surface traversée par le fluide, la masse volumique de l'eau, la profondeur qu'on veut donner à l'hydrolienne, une longueur de référence, une vitesse de référence et la viscosité dynamique de l'eau. Puis on choisit la méthode de résolution, on règle les valeurs des résidus à $10^{-5}$. Enfin avant de lancer le calcul, on définit le pas de temps, qui est choisi comme un degré de rotation (MENCHACA ROA, 2011; HALL, 2012) pour avoir le meilleur compromis entre précision et temps de calcul. On met un grand nombre de pas de temps pour atteindre une solution périodique et 100 sous-itérations par pas de temps.

\section{Résultats et discussions}

\subsection{Influence du maillage}

Pour avoir de meilleurs résultats de calcul, il faut un maillage bien adapté à la géométrie du problème. On a réalisé des simulations, avec 3 maillages différents : Le $1^{\mathrm{er}}$ maillage est le plus simple, il est non structuré. Le $2^{\text {ème }}$ maillage est structuré en partie. Le $3^{\text {ème }}$ maillage est structuré et plus fin que les deux autres (environ 180000 points). Les résultats obtenus avec ces trois maillages montrent que le résultat du troisième maillage à $T S R=2$ est le plus proche de celui obtenu par MENCHACA ROA (2011). Donc nous avons pris ce maillage pour le reste des simulations (figure 1, HAMMOUDI, 2015).

\subsection{Validation des résultats de simulation}

Comme indiqué dans l'équation (8), pour calculer le coefficient de puissance $C_{P}$, il nous faut d'abord calculer un coefficient de moment $C_{M}$, ce dernier est obtenu en prenant la moyenne d'un coefficient de moment instantané sur un tour complet. Cependant, il faut 


\section{Thème 5 - Énergies et ressources marines}

laisser se poursuivre le calcul jusqu'à l'obtention d'une solution périodique comme le montre la figure 2. Sur la figure 3, les coefficients de moment instantané de l'hydrolienne ou de chacune des pales prise individuellement, obtenus dans nos simulations, sont très proches de ceux obtenus par MENCHACA ROA (2011). Il est à noter que la figure de MENCHACA ROA (2011) présente à notre avis une erreur au niveau de sa légende, les pales 2 et 3 semblent inversées.

\subsection{Simulations avec le profil de pale NACA4415}

Après validation des simulations et du maillage utilisé, nous avons changé le profil de pale du NACA0018 projeté, au NACA4415, pour comparer la performance des deux types de profil. Les conditions de simulation sont toujours les mêmes. On a réalisé deux simulations l'une pour $T S R=1$ et l'autre pour $T S R=2$. La figure 4 illustre la différence du coefficient du moment instantané pour les deux cas, entre une hydrolienne avec des pales NACA0018 (hydrolienne 1), et une hydrolienne avec des pales NACA4415 (hydrolienne 2).

On remarque un décalage du coefficient de moment instantané entre l'hydrolienne 1 et l'hydrolienne 2. Le signal du coefficient de la première est en avance pour TSR = 1, tandis que pour $T S R=2$ il est en retard. Le moment est plus important pour l'hydrolienne 1 , laquelle a des pales projetées sur un rayon de $0.175 \mathrm{~m}$ ce qui donne une plus grande dépression entre l'intrados et l'extrados de celles-ci. Il en découle une plus grande force de portance, ainsi qu'une plus grande force tangentielle.

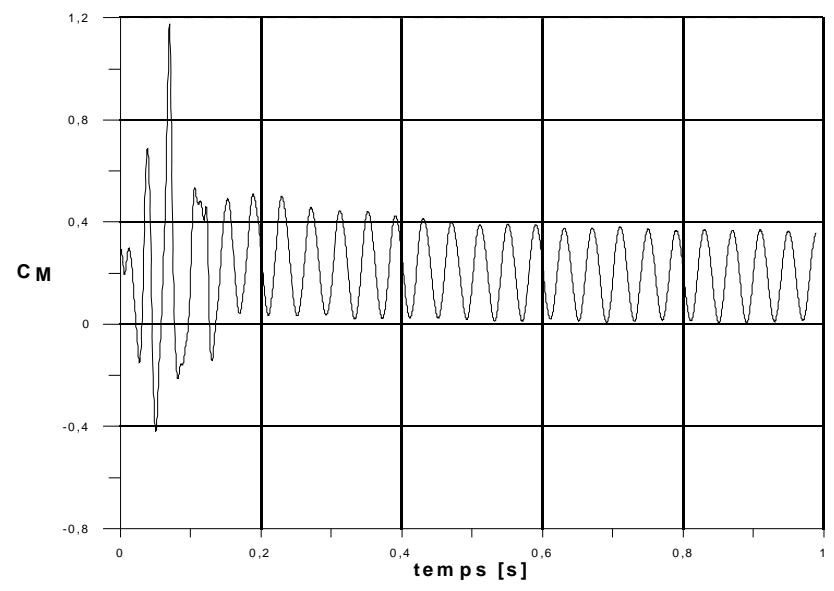

Figure 2. Solution périodique du coefficient de moment pour l'hydrolienne. 


\section{XIV èmes Journées Nationales Génie Côtier - Génie Civil \\ Toulon, 29 juin au $1^{\text {er }}$ juillet 2016}

a)
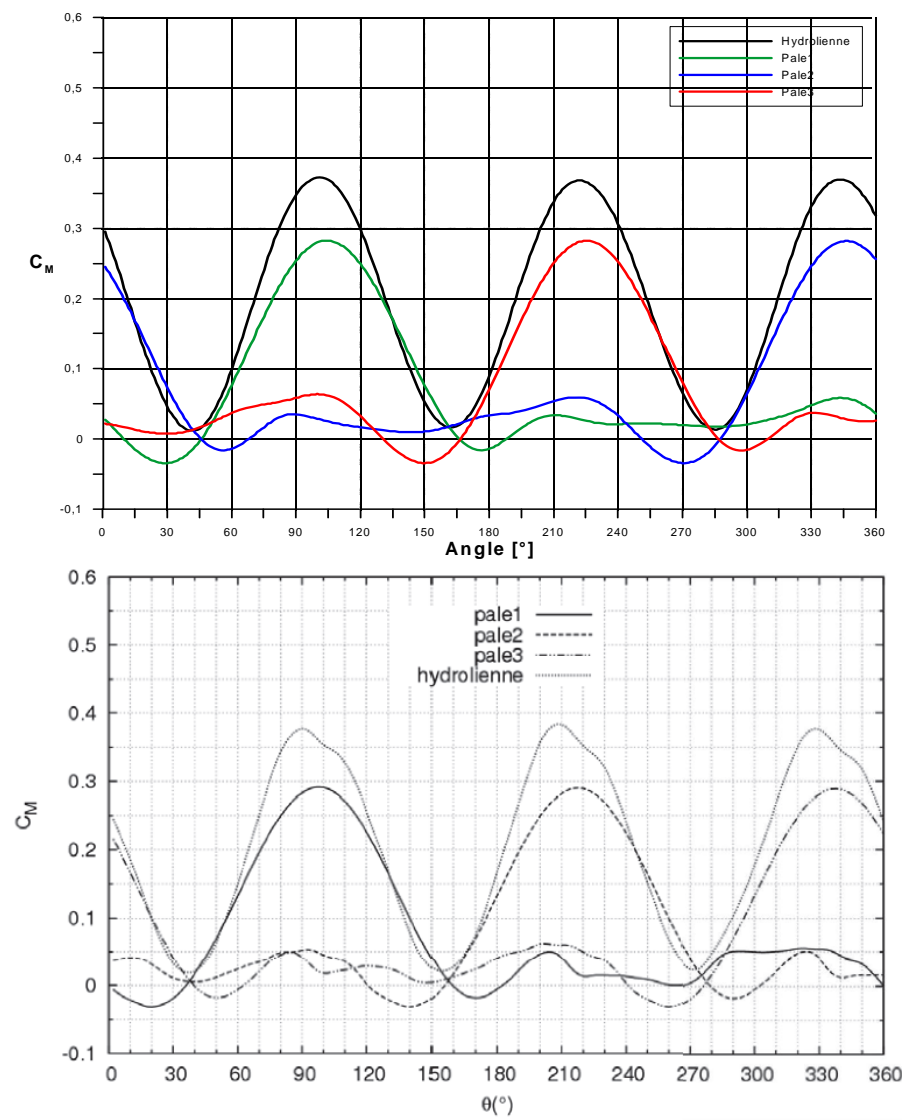

b)

Figure 3. Coefficient de moment instantané pour TSR = 2, a) notre étude, b) MENCHACA ROA (2011).
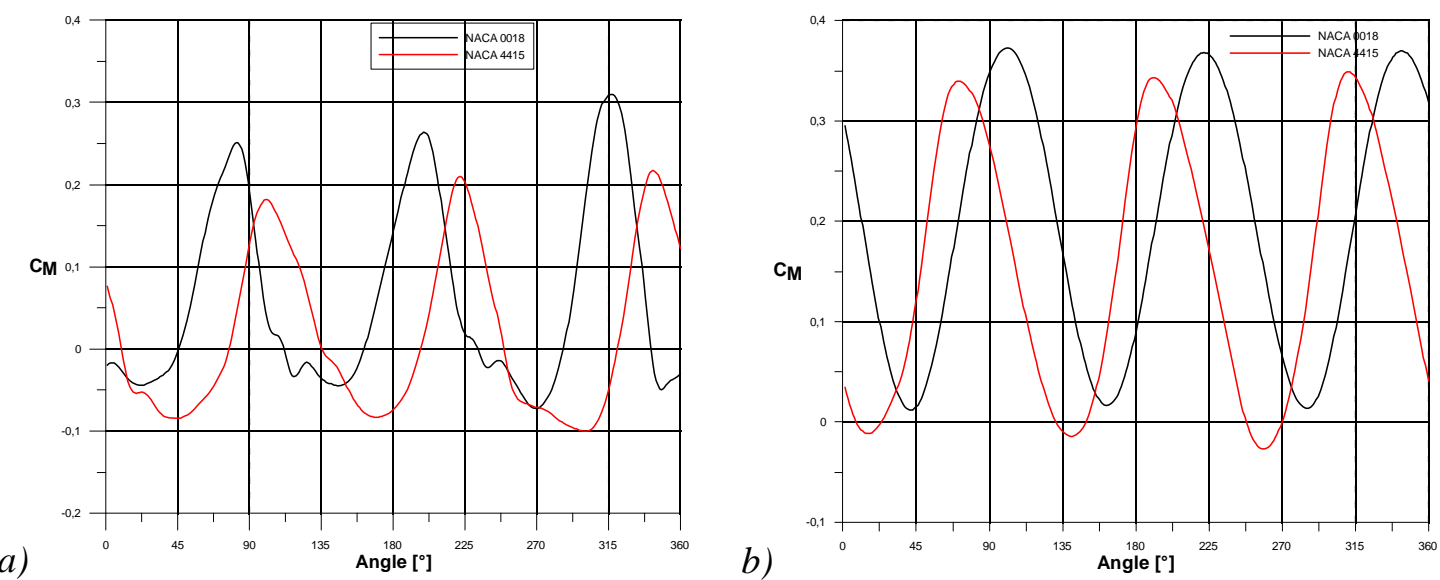

Figure 4. Coefficient de moment instantané : a) $T S R=1$, b) $T S R=2$.

\subsection{Interaction entre hydroliennes}

Pour exploiter l'énergie des courants marins, on implante des hydroliennes sous forme de fermes, similaires aux parcs d'éoliennes. Le sillage de turbulence que créent les hydroliennes vers l'aval se manifeste sous forme de charges cycliques agissant sur les 
hydroliennes situées dans cette zone et impactant leurs puissances (KERVELLA, 2014 ; MYCEK, 2014). Pour étudier cet effet d'interaction entre hydroliennes, on a effectué une simulation avec deux hydroliennes (NACA4415) alignées axialement dans le sens de l'écoulement, séparées par une distance $A=1 \mathrm{~m}$ puis $2 \mathrm{~m}$ ce qui représente respectivement environ 6 fois et 11 fois le diamètre de l'hydrolienne.

La figure 5 montre bien l'influence de la première hydrolienne sur la puissance de la deuxième. Le coefficient de moment de la deuxième hydrolienne est plus proche de celui de la première lorsque $A=2 \mathrm{~m}$, par contre pour $A=1 \mathrm{~m}$, le coefficient $C_{M H 2}$ est bien moins important que $C_{M H 1}$. La turbulence créée par la première hydrolienne se dissipe en s'éloignant d'elle, donc plus la deuxième hydrolienne est loin de la première, plus leurs coefficient de moment respectifs se rapprochent. Il y a une distance optimale entre deux hydroliennes pour laquelle leurs $C_{M}$ deviennent assez proches.

a)
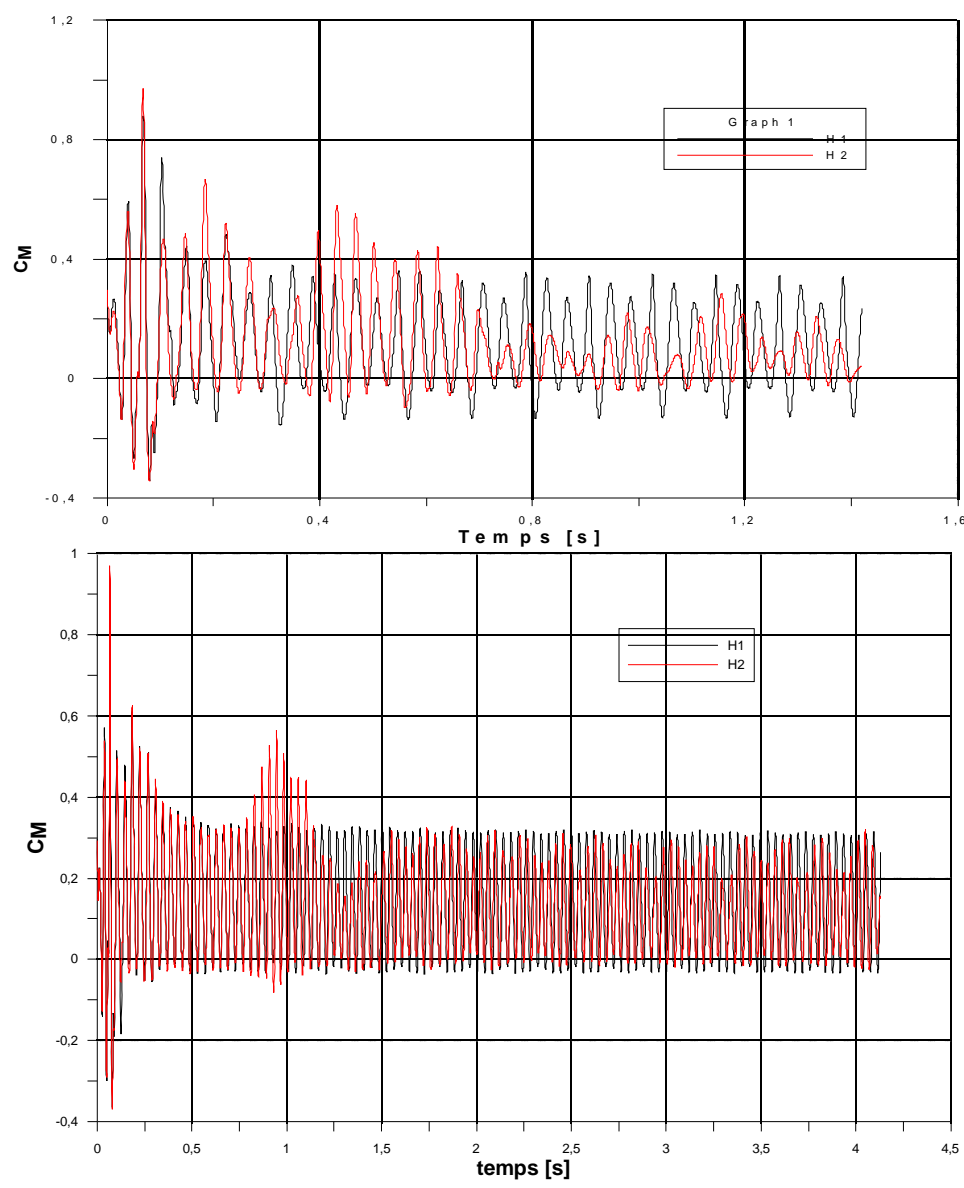

b)

Figure 5. Coefficient de moment instantané des deux hydrolienne avec :

a) $A=1 \mathrm{~m}$ et b) $A=2 \mathrm{~m}$. 


\section{XIV èmes Journées Nationales Génie Côtier - Génie Civil \\ Toulon, 29 juin au $1^{\text {er }}$ juillet 2016}

\section{Conclusions}

Dans ce travail, nous avons effectué des simulations avec ANSYS Fluent en bidimensionnel pour étudier le comportement d'une hydrolienne à axe vertical de type Darrieus avec deux types de pales d'abord le profil NACA0018 puis le profil de pale NACA 4415. Nous avons aussi abordé l'interaction entre deux hydroliennes alignées axialement. Nous avons indiqué les étapes à suivre pour la création d'une géométrie et d'un maillage en vue d'appliquer la technique du maillage glissant avec ANSYS Design Modeler, Meshing et Fluent.

Selon les simulations réalisées on remarque un décalage du coefficient de moment instantané entre l'hydrolienne 1 et l'hydrolienne 2. Le signal du coefficient de la première est en avance pour $T S R=1$, tandis que pour $T S R=2$ il est en retard. Ce résultat montre l'importance du TSR qui peut inverser le comportement pour un profil de pale donné. Pour ce qui est des interactions entre hydroliennes, les simulations avec deux hydroliennes (NACA4415) alignées axialement, séparées par une distance égale respectivement à environ 6 fois puis 11 fois le diamètre de l'hydrolienne, montrent l'effet de la première hydrolienne sur la puissance de la deuxième. Plus la deuxième hydrolienne est éloignée de la première, plus leurs coefficients de moment respectifs se rapprochent. En conclusion, la puissance d'une hydrolienne est fortement liée à la vitesse spécifique (TSR).

\section{Remerciements}

Z. Hammoudi tient à remercier l'ECAM-EPMI pour avoir financé cette étude dans le cadre de son stage de Master 2 Dynamique des fluides, Phénomènes de Transfert, Énergétique de l’UPEM - Université de Paris-Est Marne-la-Vallée. Nous remercions Monsieur Stéphane Vincent du MSME-UPEM pour ses commentaires.

\section{Références bibliographiques}

ANSYS INC (2013). ANSYS Fluent User's Guide, Release 15.0. 2620 p.

BOSSARD J. (2012). Caractérisation expérimentale du décrochage dynamique dans les hydroliennes à flux transverse par la méthode PIV - Comparaison avec les résultats issus des simulations numériques. Thèse de doctorat, université de Grenoble.

BEHRENS S., GRIFFIN D., HAYWARD J., HEMER M.A., KNIGHT C., MCGARRY S., OSMAN P., WRIGHT J. (2012). Energy Transformed and Wealth from Oceans Flagships. Ocean renewable energy, 2015-2050: an analysis of ocean energy in Australia. CSIRO. 212 p.

EDENHOFER O., PICHS-MADRUGA R, SOKONA Y, SEYBOT K., EICKEMEIER P., MATSCHOSS P., HANSEN G., KADNER S., SCHLÖMER S., ZWICKEL T., VON STECHOW C. (2011). IPCC Special Report on renewable energy Sources and Climate change Mitigation. Prepared by working Group III of the Intergovernmental 
Thème 5 - Énergies et ressources marines

Panel on Climate change. Cambridge University Press. http://dx.doi.org/10.1017/CBO9781139151153

GROUPE 12A ECP -Etudiants de l'Ecole Centrale Paris- (2005). PROJET ADEM 1 : Les hydroliennes. Rapport d'activité de découverte de l'entreprise et des métiers de l’ingénieur encadré par M. Violeau, Mme Modaressi et M. Gaudillat. Ecole Centrale Paris en collaboration avec EDF, 71 p.

HAMMOUDI Z. (2015). Modélisation et simulation d'une hydrolienne dans un courant marin. Rapport de stage Master 2 Dynamique des fluides, Phénomènes de Transfert, Énergétique, MSME-UPEM - Université de Paris-Est Marne la Valée, ECAM-EPMI Cergy-Pontoise, $34 \mathrm{p}$.

KERVELLA Y., GERMAIN G., GAURIER B., FACQ J.-V., BACCHETTI T. (2014), Mise en évidence de l'importance de la turbulence ambiante sur les effets d'interaction entre hydroliennes. XIII ${ }^{\text {èmes }}$ Journées Nationales Génie Côtier - Génie Civil, Dunkerque, pp 743-750. http://dx.doi.org/10.5150/jngcgc.2014.081

KIRKE B.K. (2011). Tests on ducted and bare helical and straight blade Darrieus hydrokinetic turbines. Renewable Energy, Vol. 36(11), pp 3013-3022. http://dx.doi.org/10.1016/j.renene.2011.03.036

MARSH P., RANMUTHUGALA D., PENESIS I., THOMAS G. (2013). Performance predictions of a straight-bladed vertical axis turbine using double-multiple stream-tube and computational fluid dynamics. J Ocean Technol., Vol. 8(1), pp 87-103.

MARSH P., RANMUTHUGALA D., PENESIS I., THOMAS G. (2015). Threedimensional numerical simulations of straight-bladed vertical axis tidal turbines investigating power output, torque ripple and mounting forces. Renewable Energy, Vol. 83, pp 67-77. http://dx.doi.org/10.1016/j.renene.2015.04.014

MENCHACA ROA A. (2011). Analyse numérique des hydroliennes à axe vertical munies d'un carénage. Thèse de Doctorat, Université de Grenoble.

MYCEK P., GAURIER B., GERMAIN G., PINON G., RIVOALEN E. (2014), Experimental study of the turbulence intensity effects on marine current turbines behaviour. Part II: Two interacting turbines. Renewable Energy, Vol. 68, pp 876-892. http://dx.doi.org/10.1016/j.renene.2013.12.048

HALL T.J. (2012). Numerical Simulation of a Cross Flow Marine Hydrokinetic Turbine. Master of Science in Mechanical Engineering, University of Washington.

JONES W.P., LAUNDER B.E. (1971). The prediction of laminarization with a twoequation model of turbulence. International Journal of Heat and Mass Transfer, Vol. 15(2), pp 301-314. http://dx.doi.org/10.1016/0017-9310(72)90076-2

TWIDELL J., WEIR T. (2006). Renewable Energy Resources, Chapter 13. Taylor \& Francis.

WILCOX D.C. (1994). Turbulence modeling for CFD, DCW Industries. 